\title{
Molecular Bose-Einstein condensation in a Bose gas with a wide Feshbach resonance at finite temperatures
}

\author{
Zeng-Qiang Yu and Lan Yin* \\ School of Physics, Peking University, Beijing 100871, China
}

(Dated: October 31, 2018)

\begin{abstract}
Bose-Einstein condensation (BEC) of Feshbach molecules in a homogeneous Bose gas is studied at finite temperatures in a single-channel mean-field approach where the Hartree-Fock energy and pairing gap are determined self-consistently. In the molecular-BEC state, the atomic excitation is gapped and the molecular excitation is gapless. The binding energy of Feshbach molecules is shifted from the vacuum value due to many-body effect. When the scattering length $a_{s}$ of atoms is negative, the system is subject to mechanical collapse due to negative compressibility. The system is stable in most regions with positive scattering lengths. However at low temperatures near the resonance, the molecular-BEC state vanishes, and the coherent mixture of atomic and molecular $\mathrm{BEC}$ is subject to mechanical collapse.
\end{abstract}

*Electronic address: yinlan@pku.edu.cn 


\section{INTRODUCTION}

Many interesting phenomena have been observed in Bose gases with Feshbach resonances. Enormous particle-loss rate due to three-body recombination was found near the resonance 1]. Oscillations between atoms and diatomic Feshbach molecules were generated by a sudden change in magnetic field [2]. Large number of Feshbach molecules were produced from Bose gases either by tuning the magnetic field through the resonance [3] or by oscillating the magnetic field at a frequency corresponding to the molecular binding energy [4]. Even tetramer molecules were created from Feshbach molecules [5]. However BEC of Feshbach molecules in Bose gases has not been achieved in experiments so far and properties of this state are waiting to be explored.

Properties of atomic-BEC state have been extensively studied theoretically [6]. Phase transition between atomic and molecular BEC was proposed near the resonance [7- 10]. However these phases suffer mechanical collapse in regions with negative scattering length

$a_{s}<0$ [11]. A recent work [12] shows that the molecular-BEC state of a Bose gas with a wide Feshbach resonance at zero temperature exists only when the atom density $n$ satisfies $n a_{s}^{3}<0.0164$ for positive scattering length $a_{s}>0$.

In this paper, the molecular-BEC state of a homogeneous Bose gas with a wide Feshbach resonance is studied at finite temperatures. In the following, we first describe the molecularBEC state in a mean-field approach where the Hatree-Fock energy and pairing gap are determined self-consistently. Long-wavelength excitations and molecular binding energy are obtained. Then mechanically stability of the molecular-BEC state is examined across the resonance, and the mean-field phase diagram is obtained. Coherent mixture of atomic and molecular BEC is also studied. Discussion and conclusion are given in the end.

\section{MEAN-FIELD THEORY OF THE MOLECULAR-BEC STATE}

\section{A. Mean-field approach}

In alkali-atom gases with Feshbach resonances, scattering states in the open channel and bound states in the closed channel are coupled together. Near a wide resonance, the effective range of the interaction is very small, and most atoms are in the open channel [13, 14]. A uniform Bose gas with a wide Feshbach resonance can be effectively described 
by a single-channel model,

$$
\mathcal{H}=-\frac{\hbar^{2}}{2 m} \psi^{\dagger} \nabla^{2} \psi+\frac{g}{2} \psi^{\dagger} \psi^{\dagger} \psi \psi-\mu \psi^{\dagger} \psi
$$

where $\psi$ is the field operator of Bose atoms in the open channel, the coupling constant is given by $g=4 \pi \hbar^{2} a_{s} / m$, and $\mu$ is the chemical potential of atoms.

In the molecular-BEC state, atoms are paired into molecules and the off-diagonal long range order (ODLRO) appears, $\Delta \equiv g\langle\psi \psi\rangle$. Without losing any generality, $\Delta \geq 0$ is assumed in the following. In the mean field, in addition to the pairing energy, both Hartree and Fock energies should be taken into account, and the mean-field Hamiltonian density is given by

$$
\mathcal{H}_{p}=\mathcal{H}_{p}^{0}-\frac{\hbar^{2}}{2 m} \psi^{\dagger} \nabla^{2} \psi+(2 g n-\mu) \psi^{\dagger} \psi+\frac{\Delta}{2}\left(\psi^{\dagger} \psi^{\dagger}+\psi \psi\right)
$$

where the constant $\mathcal{H}_{p}^{0}$ is given by $\mathcal{H}_{p}^{0}=-\left(g n^{2}+\Delta^{2} / 2 g\right)$. In this mean-field approximation, the pairing gap $\Delta$ and Hartree-Fock energy are determined self-consistently, but the fluctuation and three-body effects are ignored. The mean-field theory provides a description about single-particle excitations, but it is incapable to describe collective excitations or few-body properties such as Effimov effect. Nonetheless, the mean-field approximation can serve as a starting point of the theoretical description for the molecular-BEC state.

The mean-field Hamiltonian can be diagonalized by Bogoliubov transformation,

$$
H_{p}=\mathcal{E}_{0}+\sum_{\mathbf{k}} E_{k} \alpha_{\mathbf{k}}^{\dagger} \alpha_{\mathbf{k}}
$$

where $\alpha_{\mathbf{k}}=u_{k} \psi_{\mathbf{k}}+v_{k} \psi_{-\mathbf{k}}^{\dagger}$ is the annihilation operator of atomic quasi-particles, $E_{k}=$ $\sqrt{\left(\epsilon_{k}-\mu^{\prime}\right)^{2}-\Delta^{2}}$ is the quasi-particle energy, and the transformation coefficients are given by $u_{k}^{2}=v_{k}^{2}+1=\left[1+\left(\epsilon_{k}-\mu^{\prime}\right) / E_{k}\right] / 2$ with $\epsilon_{k}=\hbar^{2} k^{2} / 2 m$ and $\mu^{\prime}=\mu-2 g n$. The energy constant $\mathcal{E}_{0}$ is given by

$$
\mathcal{E}_{0}=\frac{1}{2} \sum_{\mathbf{k}}\left[E_{k}-\epsilon_{k}+\mu^{\prime}+\frac{\Delta^{2}}{2 E_{k}} \operatorname{coth}\left(\frac{\beta E_{k}}{2}\right)\right]-g n^{2} V,
$$

where $1 / \beta=k_{\mathrm{B}} T$ and $V$ is the volume.

The order parameter $\Delta$ can be determined self-consistently,

$$
\Delta=-\frac{g}{V} \sum_{\mathbf{k}} u_{k} v_{k}\left[1+2 f_{k}\right]
$$


where $f_{k}$ is the Bose distribution function of quasi-particles, $f_{k}=1 /\left[\exp \left(\beta E_{k}\right)-1\right]$. This self-consistency equation can be written explicitly as

$$
-\frac{m}{4 \pi \hbar^{2} a_{s}}=\int \frac{\mathrm{d}^{3} k}{(2 \pi)^{3}}\left[\frac{1}{2 E_{k}} \operatorname{coth}\left(\frac{\beta E_{k}}{2}\right)-\frac{1}{2 \epsilon_{k}}\right]
$$

where the term $-1 / 2 \epsilon_{k}$ in the integrand on the right-hand side is a counter term due to vacuum renormalization. The chemical potential $\mu$ and the order parameter $\Delta$ can be solved from Eq. (44) and the following equation for the density $n$,

$$
\begin{aligned}
n & =\frac{1}{V} \sum_{\mathbf{k}}\left[v_{k}^{2}+\left(u_{k}^{2}+v_{k}^{2}\right) f_{k}\right] \\
& =\int \frac{\mathrm{d}^{3} k}{(2 \pi)^{3}}\left[\frac{\epsilon_{k}-\mu^{\prime}}{2 E_{k}} \operatorname{coth}\left(\frac{\beta E_{k}}{2}\right)-\frac{1}{2}\right] .
\end{aligned}
$$

\section{B. Excitations in the long-wavelength limit}

There is a gap in the atomic excitation energy given by

$$
E_{0}=\sqrt{\mu^{\prime 2}-\Delta^{2}}
$$

If the molecular-BEC state is stable, one necessary condition is that the gap $E_{0}$ is real, or $-\mu^{\prime} \geq \Delta$. When $-\mu^{\prime}=\Delta$, the atomic excitation becomes gapless, $E_{0}=0$, which marks the lower boundary of the molecular-BEC phase. The upper phase boundary is located at the superfluid transition temperature $T_{p}$ where $\Delta=0$ and $E_{0}=-\mu^{\prime}$.

In addition to atomic excitations, there are collective excitations of Feshbach molecules. Although the molecular excitation energy cannot be obtained in the mean-field approximation, it can be extracted from poles of two-particle correlation functions given by

$$
\chi_{\alpha \beta}\left(\mathbf{r}-\mathbf{r}^{\prime}, \tau-\tau^{\prime}\right) \equiv-\frac{1}{\hbar}\left\langle\mathcal{T}\left[b_{\alpha}(\mathbf{r}, \tau) b_{\beta}^{\dagger}\left(\mathbf{r}^{\prime}, \tau^{\prime}\right)\right]\right\rangle,
$$

where $b_{1}=\psi^{2}, b_{2}=b_{1}^{\dagger}, b_{3}=2 \psi^{\dagger} \psi, 0 \leq \tau \leq \beta \hbar$, and $\mathcal{T}$ is the time-ordering operator. As

in the zero-temperature case [12], the correlation function can be calculated in the randomphase approximation (RPA),

$$
\chi(\mathbf{k}, \omega)=\left[1-g \chi^{(0)}(\mathbf{k}, \omega)\right]^{-1} \chi^{(0)}(\mathbf{k}, \omega),
$$

where $\chi^{(0)}(\mathbf{k}, \omega)$ is the correlation function calculated in the mean-field approximation. In RPA, the dispersion of Feshbach molecules satisfies the following equation,

$$
\operatorname{det}\left|\mathrm{I}-g \chi^{(0)}(\mathbf{k}, \omega)\right|=0
$$




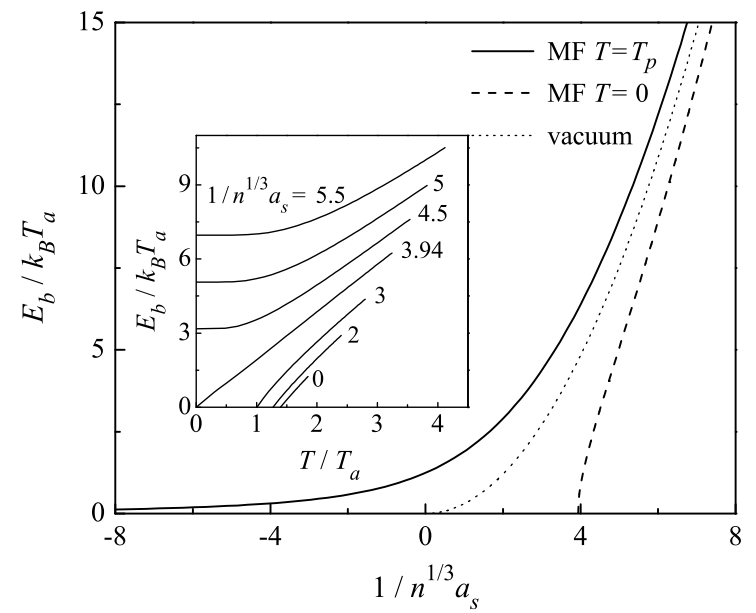

FIG. 1: Binding energy of Feshbach molecules in molecular-BEC state. The solid and dashed lines are binding energies at $T=T_{p}$ and $T=0 \mathrm{~K}$. The dotted line is the binding energy in vacuum, $E_{b 0}=\hbar^{2} /\left(m a_{s}^{2}\right)$. The inset shows the temperature dependence of the binding energy for different $n^{1 / 3} a_{s}$

where $\mathrm{I}$ is the identity matrix. The molecular excitation is gapless in the long-wavelength limit, $\mathbf{k}=0$ and $\omega=0$, following Eq. (44). At small $k$ and $\omega$, to the leading order of $k$, the molecular excitation frequency is linearly dispersed. The detail of molecular excitations will be studied in our future work.

\section{Binding energy}

The binding energy $E_{b}$ of a Feshbach molecule in the molecular-BEC state is defined as the energy difference between two atomic and one molecular excitations at $\mathbf{k}=0$. Since the molecular excitation energy is gapless in the molecular-BEC state, the binding energy of Feshbach molecules is twice the energy gap of atomic excitations, $E_{b}=2 E_{0}$. Due to many-body effect, the binding energy $E_{b}$ is different from its vacuum value $E_{b 0}=\hbar^{2} /\left(m a_{s}^{2}\right)$. At the superfluid transition temperature $T_{p}$, the binding energy is given by $E_{b}\left(T_{p}\right)=-2 \mu^{\prime}$. In the limit of weakly-attractive interaction, $n^{1 / 3} a_{s} \rightarrow 0^{-}$, we obtain

$$
E_{b}\left(T_{p}\right)=16 \pi \zeta^{-2 / 3}(3 / 2) n^{\frac{2}{3}}\left|a_{s}\right|^{2} k_{\mathrm{B}} T_{a}
$$

where $\zeta(x)$ is the Riemann-zeta function and $T_{a}$ is the ideal BEC temperature, $T_{a}=$ $\left(2 \pi \hbar^{2} / m k_{\mathrm{B}}\right)[n / \zeta(3 / 2)]^{2 / 3}$; in the limit of weakly repulsion, $n^{1 / 3} a_{s} \rightarrow 0^{+}$, the binding en- 
ergy is same as in vacuum, $E_{b}\left(T_{p}\right)=E_{b 0}=\hbar^{2} /\left(m a_{s}^{2}\right)$.

At finite temperatures, molecules not only exist for $a_{s}>0$, but also appear as looselybound pairs for $a_{s}<0$ in the mean-field theory, whereas in vacuum Feshbach molecules exist only for $a_{s}>0$. However at zero temperature, the molecular-BEC state exist only when $1 /\left(n^{1 / 3} a_{s}\right)>3.94$ [12]. In Fig. 1, the molecular binding energy in the mean-field approximation is plotted at both $T=T_{p}$ and $T=0$, in comparison with its vacuum value. The temperature dependence of the binding energy for different scattering lengths is shown in the inset. For $1 /\left(n^{1 / 3} a_{s}\right)<3.94$, the binding energy decreases monotonically with temperature, all the way to zero at the lower boundary of the molecular-BEC phase; for $1 /\left(n^{1 / 3} a_{s}\right)>3.94$, the binding energy decreases monotonically to a finite value at zero temperature. In the dilute limit, $n^{1 / 3} a_{s} \rightarrow 0^{+}$, the temperature dependence of the binding energy is weak, and the binding energy is approximately given by vacuum value $E_{b 0}$.

\section{Mechanical stability}

A mechanically stable Bose gas has positive compressibility, indicated by $\partial \mu / \partial n>0$. In the normal state, a Bose gas with $a_{s}<0$ collapses when $\partial \mu / \partial n=0$. In the Hartree-Fock approximation, this condition can be rewritten as

$$
-\frac{k_{\mathrm{B}} T_{\mathrm{C}}}{g}=\frac{1}{2} \int \frac{\mathrm{d}^{3} k}{(2 \pi)^{3}} \operatorname{csch}^{2}\left(\frac{\epsilon_{k}-\mu^{\prime}}{2 k_{\mathrm{B}} T_{\mathrm{C}}}\right) .
$$

The collapse temperature $T_{\mathrm{C}}$ is always higher than the molecular BEC transition temper-

ature $T_{p}\left[15\right.$-17]. In the limit of weakly-attractive interaction, $n^{1 / 3} a_{s} \rightarrow 0^{-}$, the collapse temperature $T_{\mathrm{C}}$ approaches to the ideal atomic BEC temperature $T_{a}$,

$$
T_{\mathrm{C}}=T_{a}\left[1+\frac{16 \pi}{3 \zeta^{4 / 3}\left(\frac{3}{2}\right)} n^{1 / 3}\left|a_{s}\right|\right] .
$$

In contrast, a normal Bose gas with $a_{s}>0$ is always mechanically stable.

We examine the compressibility of the molecular-BEC state numerically following Eq. (4) and (5). In Fig. 2, the chemical potential $\mu$ is plotted as a function of density $n$ at given temperatures and scattering lengths. For $a_{s}<0$, the chemical potential $\mu$ is monotonically decreasing with the increase of $n, \partial \mu / \partial n<0$, showing that the system is mechanically unstable as found in previous studies [15, 16]. For $a_{s}>0$, the compressibility is always positive, $\partial \mu / \partial n>0$, and the molecular-BEC state is mechanically stable. Therefore, in a 

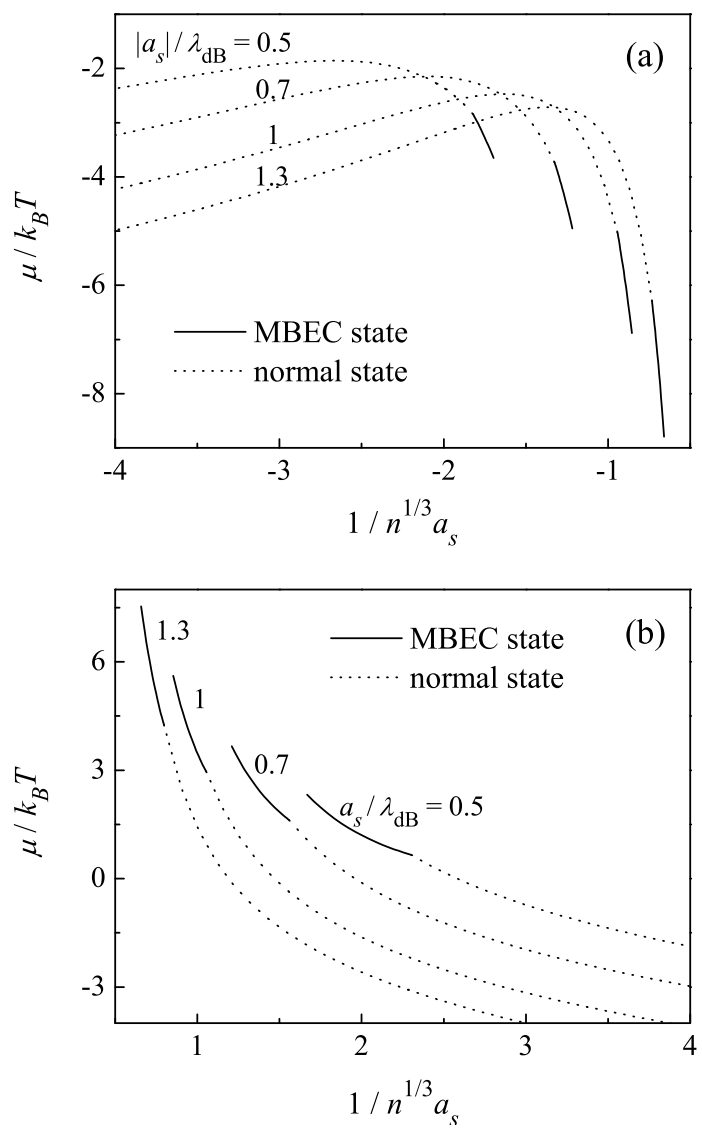

FIG. 2: Chemical potential $\mu$ versus $1 / n^{1 / 3} a_{s}$. The solid line is the chemical potential in the molecular-BEC (MBEC) state and the dotted line is the chemical potential in the normal state, (a) with attractive interaction, $a_{s}<0$; (b) with repulsive interaction, $a_{s}>0$. The lines with $\left|a_{s}\right| / \lambda_{\mathrm{dB}}=0.5,0.7,1.0$, and 1.3, are plotted, where $\lambda_{\mathrm{dB}}=\sqrt{2 \pi \hbar^{2} / m k_{\mathrm{B}} T}$ is the thermal de Broglie wavelength.

uniform Bose gas, the molecular-BEC state can only exist with positive scattering lengths, $a_{s}>0$. In a trap, the molecular-BEC state may be stabilized by the finite-size effect, similar to trapped BEC with attractive interactions [18].

\section{E. Mean-field phase diagram}

The upper boundary of the molecular-BEC phase is determined by the disappearance of order parameter $\Delta=0$, corresponding to the second-order phase transition between the normal and molecular-BEC phases. At the transition temperature $T_{p}$, Eq. (4) is the same 
as the Thouless criterion which marks the pairing instability of the normal phase,

$$
-\frac{1}{g}=\int \frac{\mathrm{d}^{3} k}{(2 \pi)^{3}}\left[\frac{1}{2\left(\epsilon_{k}-\mu^{\prime}\right)} \operatorname{coth}\left(\frac{\epsilon_{k}-\mu^{\prime}}{2 k_{\mathrm{B}} T_{p}}\right)-\frac{1}{2 \epsilon_{k}}\right] .
$$

The density equation (5) at $T_{p}$ becomes the Hartree-Fock self-consistency condition of the normal Bose gas

$$
n=\int \frac{\mathrm{d}^{3} k}{(2 \pi)^{3}} \frac{1}{e^{\left(\epsilon_{k}-\mu^{\prime}\right) / k_{\mathrm{B}} T_{p}}-1} .
$$

In the limit of the weakly-attractive interaction, $n^{1 / 3} a_{s} \rightarrow 0^{-}$, the transition temperature $T_{p}$ is close to the ideal atomic BEC temperature $T_{a}=\left(2 \pi \hbar^{2} / m k_{\mathrm{B}}\right)[n / \zeta(3 / 2)]^{2 / 3}$,

$$
T_{p}=T_{a}\left[1+\frac{8 \pi}{3 \zeta^{4 / 3}\left(\frac{3}{2}\right)} n^{1 / 3}\left|a_{s}\right|\right],
$$

as found in a previous study [17].

In the opposite limit, $n^{1 / 3} a_{s} \rightarrow 0^{+}$, molecules are tightly bound with the binding energy approximately given by $E_{b 0}$. From Eq. (12), the chemical potential is approximately given by $\mu=-E_{b 0} / 2$. The solution of density equation (13) yields

$$
k_{\mathrm{B}} T_{p}=E_{b 0} \ln ^{-1}\left(E_{b 0} / k_{\mathrm{B}} T_{a}\right) / 3
$$

which is essentially the same as the mean-field result about the BEC limit of a Fermi gas with a BEC-BCS crossover. As pointed out in earlier works [19], the divergent mean-field transition temperature in this limit in fact corresponds to the temperature of molecule dissociation, not molecular condensation. The transition temperature $T_{p}$ can be renormalized by pairing fluctuations [20].

The lower boundary of the molecular-BEC phase is determined by $\Delta=-\mu^{\prime}$, where the excitation energy becomes gapless, $E_{k}=\sqrt{\epsilon_{k}\left(\epsilon_{k}-2 \mu^{\prime}\right)}$. At zero temperature, this boundary is located at $1 /\left(n^{1 / 3} a_{s}\right)=4(3 / \pi)^{1 / 3} \simeq 3.94$ [12]. Disappearance of gap in excitation energies was interpreted as appearance of atomic condensation [15, 20]. However, as discussed later in section III, a coherent mixture with atoms and molecules is mechanically unstable.

The order parameter $\Delta$ in the molecular-BEC phase evolves smoothly between two phase boundaries. Based on the above results, the mean-field phase diagram is plotted in Fig. 3. The molecular-BEC phase is labeled as $\mathrm{P}(\mathrm{S})$ for $a_{s}>0$ and $\mathrm{P}(\mathrm{C})$ for $a_{s}<0$, where symbols (S) and (C) represent mechanical stability and mechanical collapse. 


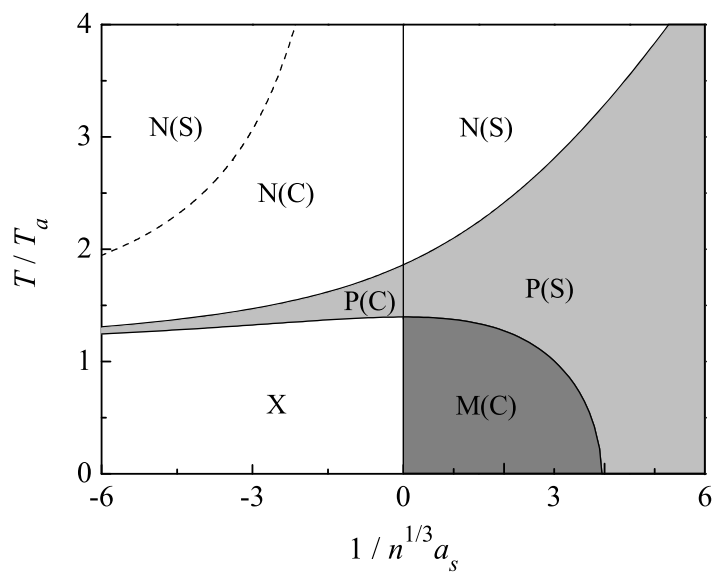

FIG. 3: Mean-field phase diagram of a pairing Bose gas with a wide Feshbach resonance. P(S) denotes the stable molecular-BEC phase; $\mathrm{P}(\mathrm{C})$ denotes the collapsing molecular-BEC phase; N(S) denotes the stable normal phase; $\mathrm{N}(\mathrm{C})$ denotes the collapsing normal phase; $\mathrm{M}(\mathrm{C})$ denotes the collapsing coherent mixture of atoms and molecules; X denotes no solution according to the meanfield theory. The Dashed line is the mean-field collapse temperature of the normal phase with attractive interactions.

Recently similar phase diagrams were obtained in Ref. [20] within the approach pioneered by Nozières and Schmitt-Rink (NSR) [21]. In the NSR approach, pairing fluctuations are treated by the $t$-matrix approximation, and the total particle density includes not only the mean-field density, but also the density of thermal molecules. Although there are some discrepancies, most features of our mean-field phase diagram Fig. 3 qualitatively agree with those in Ref. [20]. The condensation temperature of the MBEC phase is significantly reduced in the NSR approach. In the limit of weakly-repulsive interaction, it can recover the condensation temperature of an ideal molecular Bose gas, $T_{p}=T_{a} / 2^{5 / 3}$. Both NSR and mean-field phase diagrams show that there is a transition from the molecular BEC to the mixture state. As shown in the next section, the mixture state is mechanically unstable in the mean-field approximation. In Ref. [20], the normal state was found to be stable in the simple NSR approach, but unstable close to the resonance in the extended NSR approach by taking into account interaction between molecules . In the mean-field approach, the normal state is unstable near the resonance only on the attractive-interaction side. 


\section{MECHANICAL COLLAPSE OF COHERENT ATOM-MOLECULE MIX- TURE}

At the lower boundary of the molecular-BEC phase, the atomic excitation energy vanishes, $E_{0}=0$, suggesting the appearance of the coherent atom-molecule mixture. In the mixture, since both molecules and atoms exhibit ODLRO, two order parameters can be introduced [12], $\psi_{0} \equiv\langle\psi\rangle$ describing atomic condensate and $\Delta \equiv g\langle\delta \psi \delta \psi\rangle$ describing molecular condensate, where $\delta \psi \equiv \psi-\psi_{0}$. These two order parameters are in principle independent, whereas in the atomic-BEC state $\Delta$ is a function of $\psi_{0}$. In the mean-field approximation, the Hamiltonian density for this mixture state is given by

$$
\begin{aligned}
\mathcal{H}_{m}=\mathcal{H}_{m}^{0} & -\frac{\hbar^{2}}{2 m} \delta \psi^{\dagger} \nabla^{2} \delta \psi+(2 g n-\mu) \delta \psi^{\dagger} \delta \psi \\
& +\frac{1}{2}\left[\left(\Delta+g \psi_{0}^{2}\right) \delta \psi^{\dagger} \delta \psi^{\dagger}+\text { h.c. }\right],
\end{aligned}
$$

where $\mathcal{H}_{m}^{0}=g n_{0}^{2} / 2-\mu n_{0}-g \delta n^{2}-|\Delta|^{2} / 2 g, n_{0} \equiv\left|\psi_{0}\right|^{2}$ is the atomic-condensate density, $\delta n \equiv\left\langle\delta \psi^{\dagger} \delta \psi\right\rangle$, and $n=n_{0}+\delta n$. The order parameter $\psi_{0}$ should minimize the mean-field thermodynamic potential, which leads to the saddle point condition

$$
\mu=g\left(n_{0}+2 \delta n\right)+\Delta \frac{\psi_{0}^{*}}{\psi_{0}} .
$$

To satisfy the saddle point condition, the product $\Delta \psi_{0}^{2}$ must be real. For simplicity, we choose $\Delta>0$.

As in the molecular BEC case, the mean-field Hamiltonian of the mixture can be diagonalized by Bogoliubov transformation, where the field operator of atomic quasi-particles is given by $\alpha_{\mathbf{k}}=u_{k} \psi_{\mathbf{k}}+v_{k} \psi_{-\mathbf{k}}^{\dagger}, u_{k}^{2}=v_{k}^{2}+1=\left[1+\left(\epsilon_{k}-\mu^{\prime}\right) / E_{k}\right] / 2$, and the quasi-particle energy

is given by $E_{k}=\sqrt{\left(\epsilon_{k}-\mu^{\prime}\right)^{2}-\left(g \psi_{0}^{2}+\Delta\right)^{2}}$. The order parameter $\Delta$ can be determined by the self-consistency equation

$$
\Delta=-\frac{g}{V} \sum_{\mathbf{k}} u_{k} v_{k}\left[1+2 f_{k}\right]
$$

i.e.

$$
-\frac{m}{4 \pi \hbar^{2} a_{s}}+\frac{\psi_{0}^{2}}{\Delta+g \psi_{0}^{2}}=\int \frac{\mathrm{d}^{3} k}{(2 \pi)^{3}}\left[\frac{1}{2 E_{k}} \operatorname{coth}\left(\frac{\beta E_{k}}{2}\right)-\frac{1}{2 \epsilon_{k}}\right]
$$

where similar to Eq. (4) the last term in the integrand is the counter term. The equation for the total density is given by

$$
n=n_{0}+\int \frac{\mathrm{d}^{3} k}{(2 \pi)^{3}}\left[\frac{\epsilon_{k}-\mu^{\prime}}{2 E_{k}} \operatorname{coth}\left(\frac{\beta E_{k}}{2}\right)-\frac{1}{2}\right] .
$$




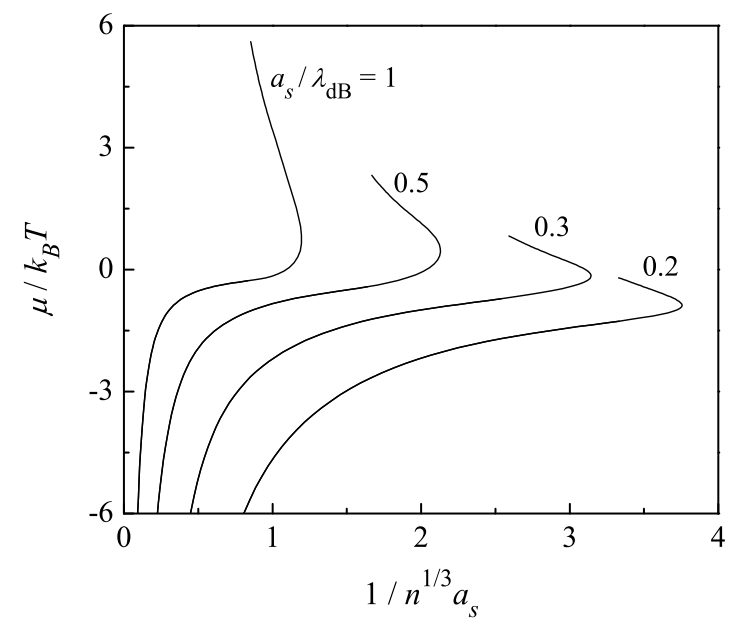

FIG. 4: Chemical potential $\mu$ of the coherent mixture versus $1 / n^{1 / 3} a_{s}$ with $a_{s} / \lambda_{\mathrm{dB}}=0.2,0.3,0.5$ and 1.0 respectively, where $\lambda_{\mathrm{dB}}=\sqrt{2 \pi \hbar^{2} / m k_{\mathrm{B}} T}$.

From the saddle point condition Eq. (17), the ratio $\psi_{0}^{*} / \psi_{0}$ is either 1 or -1 , and the quasi-particle excitation energy can be rewritten as

$$
E_{k}=\sqrt{\left(\epsilon_{k}+2 g n_{0}\right)\left(\epsilon_{k}-2 \Delta \psi_{0}^{*} / \psi_{0}\right)}
$$

If the excitation energy $E_{k}$ is real, two conditions, $g n_{0}>0$ and $\psi_{0}^{*} / \psi_{0}=-1$, must be true, which cannot be satisfied when the scattering length $a_{s}$ is negative, indicating that the mixture cannot exist with $a_{s}<0$. When $\psi_{0}=0$, there is a transition from the coherent mixture phase to the molecular-BEC phase. At zero temperature, the transition point between the two phases locates at $1 /\left(n^{1 / 3} a_{s}\right) \simeq 3.94$.

For fixed total density, the chemical potential and order parameters can be solved from Eq. (17, 18, 19). The solution shows a reentrant behavior near the transition to the molecularBEC phase, as shown in Fig. 4. The reentrance range in temperature is broadened close to the resonance. This reentrance behavior is likely to be an unphysical result in the mean-field approximation near the transition, similar to that in the Popov approximation of a Bose gas near the atomic BEC temperature [22]. Away from this reentrance, as shown in Fig. 4, the chemical potential $\mu$ is a monotonically decreasing function of density $n$ for fixed $T$ and $a_{s}, \partial \mu / \partial n<0$, meaning that compressibility is negative. Therefore the coherent mixture is subject to mechanical collapse. 


\section{DISCUSSION AND CONCLUSION}

In the mean-field approximation, although the pairing gap and Hartree-Fock energy are computed self-consistently, fluctuations are ignored. In the NSR approach [20], the pairing fluctuation is treated, which significantly reduced the condensation temperature. However, the Hartree-Fock energy is ignored in the NSR approach, affecting the condition of mechanical stability. In future many-body theories, the self-energy should be determined selfconsistently with fluctuations properly treated. Experimentally, three-body recombination

causes enormous particle-loss near the resonance. In a more accurate microscopic theory, both many-body and few-body effects should be considered. Nonetheless, the mean-field theory provides a basic picture about the strongly interacting Bose gas, with most features of the phase diagram in qualitative agreement with the NSR approach [20].

In conclusion, the molecular-BEC state of a homogeneous Bose gas with a wide Feshbach resonance is studied at finite temperatures. In the long-wavelength limit, the atomic excitation is gapped and molecular excitation is gapless. The molecular binding energy is changed from its vacuum value due to many-body effect and can even vanish when the scattering length $a_{s}$ is finite. When $a_{s}<0$, the compressibility of the molecular BEC state is negative and the system is subject to mechanical collapse. The possibility of coherent atom-molecule mixture is also explored, but the compressibility of the mixture is negative and the mixture is subject to mechanical collapse. Based on these results, a mean-field phase diagram at finite temperatures is obtained. This work is supported by NSFC under Grant No. 10674007 and No. 10974004, and by Chinese MOST under grant number 2006 CB921402.

[1] J. Stenger, S. Inouye, M. R. Andrews, H. J. Miesner, D. M. Stamper-Kurn, and W. Ketterle, Phys. Rev. Lett. 82, 2422 (1999).

[2] E. A. Donley, N. R. Claussen, S. T. Thompson, and C. E. Wieman, Nature 417, 529 (2002).

[3] K. Xu, T. Mukaiyama, J. R. Abo-Shaeer, J. K. Chin, D. E. Miller, and W. Ketterle, Phys. Rev. Lett. 91, 210402 (2003).

[4] S. T. Thompson, E. Hodby, and C. E.Wieman, Phys. Rev. Lett. 95, 190404 (2005).

[5] F. Ferlaino, S. Knoop, M. Berninger, W. Harm, J. P. D'Incao, H.-C. Nägerl, and R. Grimm, Phys. Rev. Lett. 102140401 (2009). 
[6] For reviews see, A. Griffin, Excitations in a Bose-Condensed Liquid (Cambridge University Press, New York, 1993); F. Dalfovo, S. Giorgini, L. P. Pitaevskii and S. Stringari, Rev. Mod. Phys. 71, 463 (1999); A. J. Leggett, Rev. Mod. Phys. 73, 307 (2001).

[7] L. Radzihovsky, J. Park, and P. B. Weichman, Phys. Rev. Lett. 92, 160402 (2004).

[8] M. W. J. Romans, R. A. Duine, S. Sachdev, and H. T. C. Stoof, Phys. Rev. Lett. 93, 020405 (2004).

[9] Y.-W. Lee and Y.-L. Lee, Phys. Rev. B 70, 224506 (2004).

[10] E. Braaten and D. Zhang, Phys. Rev. A 75, 063624 (2007).

[11] S. Basu and E. J. Mueller, Phys. Rev. A 78, 053603 (2008).

[12] L. Yin, Phys. Rev. A 77, 043630 (2008).

[13] S. Simonucci, P. Pieri, and G. C. Strinati, Europhys. Lett. 69, 713 (2005).

[14] L. Yin and Z.-H. Ning, Phys. Rev. A 68, 033608 (2003); Y. Zhang and L. Yin, Phys. Rev. A 72,043607 (2005).

[15] H. T. C. Stoof, Phys. Rev. A 49, 3824 (1994).

[16] E. J. Mueller and G. Baym, Phys. Rev. A 62, 053605 (2000).

[17] G. S. Jeon, L. Yin, S. W. Rhee, and D. J. Thouless, Phys. Rev. A 66, 011603(R) (2002).

[18] C. C. Bradley, C. A. Sackett, and R. G. Hulet, Phys. Rev. Lett. 78, 985 (1997).

[19] C. A. R. Sá de Melo, M. Randeria, and J. R. Engelbrecht, Phys. Rev. Lett. 71, 3202 (1993).

[20] A. Koetsier, P. Massignan, R. A. Duine, and H. T. C. Stoof, Phys. Rev. A 79, 063609 (2009).

[21] P. Nozières and S. Schmitt-Rink, J. Low Temp. Phys. 59, 195 (1985).

[22] J. O. Andersen, Rev. Mod. Phys. 76, 599 (2004). 